\title{
Hope in older women across cultures
}

\begin{abstract}
The article presents a cross-sectional study, based on the hope questionnaire developed by Herth. ${ }^{1}$ The main aim of the study was to find out the meaning that hope may assume in later life, and particularly in women. The participants of the study were eight women in their late sixties, coming from two cultural backgrounds: UK and Italy. Their different backgrounds have given me a valuable opportunity to look into possible cultural variables that may have had an impact on their perception of hope and the meaning that they attribute to it. The participants' hope-narratives have been explored within an integrative theoretical model of hope, which takes account of key hope-fostering factors for aging well, namely perceived or predicted physical and psychological wellbeing that can facilitate a sense of agency within relational contexts. The findings emerged from the participants' accounts, clearly reveal the complex aspects of hope and call for a holistic revisiting of this phenomenon within an integrative construct that can reflect its multidimensional often contradictory essence.
\end{abstract}

Volume 3 Issue 2 - 2019

\author{
Sonia Gallucci \\ Regent's Institute of Languages and Culture, Regent's University \\ London, UK
}

\begin{abstract}
Correspondence: Sonia Gallucci, Regent's Institute of Languages and Culture, Regent's University London, Inner Circle - Regent's Park, London NWI 4NS, UK, Tel +44(0)207877505, Email galluccis@regents.ac.uk
\end{abstract}

Received: February 06, 2019 | Published: March 20, 2019

Keywords: hope, older adults, later life

\section{Introduction}

My interest in this topic comes primarily from the frequency that over the years I have heard people using the term 'hope' in various contexts across cultures. In the last three years, I have become increasingly interested in the meaning of hope and what it means particularly for older women, as I have been witnessing my mother carrying on with her life of physical and emotional struggles since my father passed away, after fifty years of marriage. "Speriamo" (I hope/ let's hope so) she would say repeatedly, while her physical disability (being disabled with a degenerative joint condition) would not let her sleep, nor stand or sit comfortably. "Speriamo" she would say every time the emptiness for the loss of my father seems unbearable to her. "Speriamo che domani vada meglio" (I hope/Let's hope that tomorrow will go better). With the personal firm conviction that it is this hope that gives her the strength to carry on with her life day after day, despite the odds and the pain, I decided to write this paper, in order to find out the meaning that older adults, and particularly women, attribute to hope, and whether such meaning is shaped by their life experiences and by their cultural backgrounds.

\section{Methodology}

The participants of this study were eight women over the age of 65. Four of them come from the U.K. and the other four are from Italy. The different socio- political and national backgrounds of my participants have given me a valuable opportunity to look into possible cultural variables that may have had an impact on their perception of hope and the meaning that they attribute to it. After being made aware of the ethical issues involved in this study, all participants provided written consent to share their answers with a larger audience for academic purposes. To protect their identities, the pseudonyms of Ilenia, Carlotta, Pierina, Giovanna, Gwen, Jacqui, Mo and Nicole have been used throughout the paper.

The primary data of this study have been gathered through a questionnaire. More specifically, I have asked my participants to answer in writing to six open questions related to hope. The all did it either by email or through the online application WhatsApp; except for Ilenia, who could not write due to a severe form of arthritis, hence, she provided her answers through a video-call that I made via the online application Skype. The six questions have been adapted from the hope questionnaire developed by Herth ${ }^{1,2}$ and for the Italian participants they have also been translated. The data supporting the findings of this study are available within its supplementary materials.

\section{An integrative view on hope}

Hope can firstly be understood in its semantic connotations as a noun "a feeling of expectation and desire for a particular thing to happen" and as a verb "want something to happen or be the case" (www.en.oxforddictionaries.com/definition/hope). The mutual relationship between the verbal and substantive construct of the term, as stated by Eliott \& Olver, ${ }^{3}$ seems to highlight the extent to which hope can be internalised in one's existence (as an emotion or as an action), or conversely be objectified as just being 'out there'. It is inbetween these two existential polarities that hope often emerges and manifests itself in a myriad of modalities that reflect the complexity of its meaning.

Indeed, there seem to be a general agreement across psychotherapeutic traditions, that hope needs to be considered as a "multidimensional, often contradictory, construct" that comprises psychological, physical, social and spiritual aspects. ${ }^{4}$ It also appears to be essential to view it as a dynamic process, rather than as a fixed entity. A process inclined to change over time depending on individuals' cognitive and emotional responses to life experiences. ${ }^{5}$ Within this multidimensional paradigm, Dufault \& Martocchio ${ }^{6}$ identifies six spheres of hope, those are: affective, cognitive, behavioural, affiliative, temporal and contextual. Particular emphasis seems to be put on the cognitive and behavioural spheres, as they would represent a fertile ground for action, be it psychological, physical, social or spiritual. In their view, such action would aim to the achievement of positive global and individual outcomes, primarily projected in the future. Future-oriented thinking together with a positive attitude and a willingness to achieve those outcomes seem to be salient ingredients in hopeful individuals. ${ }^{7}$ These factors mirror the key features that Snyder, ${ }^{8,9}$ the founder of positive psychology, identified in his wellknown studies on hope in psychotherapy.

These are: goals, pathways and agency. He considers hope to be mainly a way of thinking, with feelings being influential but not predominant. To put it into Snyder's words, ${ }^{9}$ hope is a "motivational state based on an interactively derived sense of successful (a) agency 
(goal-directed energy) and (b) pathways (planning to meet goals)" (p. 250). Hence, in his view the perception of the achievement of such goals shapes individuals' hopeful thinking and this, in turn, would condition their emotions. Snyder's pragmatic views on hope are shared by many mental health practitioners, particularly by those who privilege a cognitive behavioural approach to therapy. ${ }^{10,11}$ This approach implies an appraisal-like process that can foster hope, with a view of enhancing individuals' life towards emotion selfregulation $^{12}$ and self-actualisation. ${ }^{11,13}$ As Vande Kemp, ${ }^{14}$ indicates, this is also a process that entails "inner readiness" from its recipients (p. 30), and a capacity for relatedness and for love where hope can be generated and nurtured. In his view, hope is fundamentally relational and can be engendered by three main factors: these are faith (outer, inner or divine), memory (as an internal form of actualised hope) and fantasy (or imagination for creative meaning). Vande Kemp's views can be associated with Jung's ${ }^{15}$ stance on hope as one of the highest achievements of human efforts. Indeed, in Jung's view, hope (together with love, faith and insight) represents a crucial component for reaching spiritual and psychic wellbeing. It is a relational hope that influences and it is influenced by life experiences (including events and people). On the whole, a capacity for concrete and symbolic thinking (shaped by experience) together with an ability to reach out to inner and outer resources, that can lead to action within relational contexts, appear to be key features for an overarching theoretical model of hope in integrative psychotherapy. However, as detailed below, when dealing with older adults, additional factors need to be taken in to careful consideration.

\section{Hope in later life}

When working therapeutically with older adults it is important to consider additional factors that can influence their perception of hope and the meaning that they attach to it. Bergin \& Walsh, ${ }^{5}$ for instance, highlight the importance of looking not only at future, but also at present and past achievements as a way to facilitate hope in later life. Indeed, depending on clients' needs, hope can be fostered by looking at the future, through the setting up of feasible pathways to achieve realistic goals ${ }^{8}$ at the present by emphasising how reaching a long life, and surviving the multiple losses that older adults are likely to have experienced, can indeed be appreciated as a great life achievement; finally, hope could also be engendered by looking at the past. The latter is called by Bergin \& Walsh ${ }^{5}$ the 'work of despair' and it is probably the most heart-breaking form of work with older adults, who looking at the past can find no memories that deserve to be cherished; hence, the despair. In this case, hope could be facilitated in therapy by focussing on the achievement of a good enough ending, be it the acknowledgment of their last wishes or the planning of their own death, that once accomplished could ern the right to be held in the memories of others, including those of the therapist.

Memory emerges to be a crucial component of hope at any age, but it seems to assume a particular importance for adults in later or end of life, as it takes the shape of "legacy to some or someone who is willing to listen" $"$ henceforth, an insightful source of hope for the generations that we leave behind. ${ }^{16}$ Being able to share life memories through narratives, has been observed to be an important factor across a variety of studies about hope in later life. ${ }^{1,7,17}$ For instance, in Olsen's ${ }^{7}$ study with older adults in long-term care, narratives and life experiences, alongside relationships, spirituality and goaldevelopment, emerged to be invaluable sources of hope. Particularly, self-narratives seem to be a significant way of connecting with own strengths and coping mechanisms experienced in the past, while at the same time developing self-reflection and resilience.

Uplifting memories and interconnectedness have been identified as hope-fostering categories also by Herth ${ }^{1}$ (in his study with older adults within communities and institutional settings. Other factors that seem to be salient in older hopeful thinkers are a personal inclination to be humorous and light-hearted, a spiritual or philosophical stance towards life and a willingness to pursue activities, by applying cognitive strategies (for the affirmation of worth) with attainable aims. ${ }^{1,2}$ A spiritual base that can be shared with others (including religious, philosophical or collective entities) seems also to emerge as a crucial component of hope in later or end of life. To put it into Szasz's ${ }^{18}$ words, spirituality, and particularly when based on religious faith, could indeed be "one of the best investments than one can make", since it would give some sort of "hope for the hopeless" (p. 287).

Along with the psychological and social hope-fostering factors mentioned above, we also ought to consider physiological elements that can play an important role in engendering hope in later life. Indeed, as Wroblesky \& Snyder ${ }^{17}$ point out, as life goes on, together with interpersonal losses (such as the death of loved ones) we are more prone to experience biological losses, as we see the "decline in physical health and in cognitive functioning" (p. 219). Thus, a higher sense of hope has been observed in older adults who have a relatively good health, or a perceived general physical wellbeing. This biological factor is linked to feelings of self-efficacy, accomplishment, enthusiasm, confidence and ultimately to a general sense of life satisfaction, which is considered to be one of the main factors for aging well. According to Wroblesky \& Snyder,${ }^{17}$ being able to be active agents in the achievement of own goals at later stages of life, implies an element of external (or physical) and internal (or emotional) control, which seems to be the gatekeeper for hopeful thinking, as it would allow individuals to maintain a sense of agency in the face of their losses. Hence, when working with adults in later life, it is essential to take into careful consideration a multitude of factors that can foster (or inhibit) their hope and shape their lived experiences as life goes on. Particularly, physical and psychological wellbeing that can facilitate a sense of agency within relational contexts (tangible and spiritual) seem to emerge as key hope-fostering factors for aging well.

\section{Participants' hope}

A thematic analysis of my primary data revealed that the above key hope- fostering categories emerged to various degrees from all participants' accounts. They all showed future-oriented hopeful thinking in the achievement of their goals, on a personal level (e.g. good health for self and family) and on a more general scale (e.g. peace in the world). When confronted with the question "what kind of things do you do to help yourself regain hope?", active agency strongly emerged from the British participants, as they would look for feasible pathways while envisaging hope-restoration as a more immediate goal. ${ }^{8}$ So, for instance, Gwen, Mo and Jacqui would carry out enjoyable activities, such as going for walks, watching funny movies or talking to friends, whereas Nicole and three of the Italian participants (Carlotta, Ilenia and Pierina) would turn to their spiritual side and pray. The reliance on a spiritual source of hope, can be linked to the importance that some individuals attribute to religious faith, as a sheltered hope-provider when a sense of hopelessness is perceived. ${ }^{18}$ 
Faith (be it inner, outer or divine) emerged as an important hopefostering category among all participants. ${ }^{14}$ However, while religious faith emerged to be a secure source of hope particularly for the participants from Italy, for the British participants hope seems to rely more on their here-and-now physical (rather than transcendental) surroundings, including people and activities, and on a "c'est la vie" (Mo, 5.) attitude towards life. In this sense, pragmatic versus fatalistic views on life could reflect the different degrees of hope that participants may experience according to their cultural heritage (including religious and philosophical beliefs).

A perceived or predicted sense of physical wellbeing for self and loved ones, has also been indicated by participants as a significant hope-endangering factor. ${ }^{17}$ It is health for themselves that the participants wish for, since "if there is health there is everything" (Ilenia 1.); and it is also good health for family members "and people [they] love" (Pierina 1.). Physical health is considered by participants to be so vital that when it is lacking, can hinder their capacity for hopeful thinking, and even lead to despair. As in Giovanna's case, who "saw dark" (5.) when she witnessed the physical deterioration of her husband before he died.

A further category that seem to foster hope in all participants is the cognitive ability to think positively; an ability linked to optimism and shaped by a recognised capacity to overcome personal (often tragic) life struggles. As in the case of Jacqui when she had cancer, hope and determination to live was restored by a personal inclination of being 'optimistic' and by the experiential outcome of being healthy again. As she stated, "I have always been an upbeat and optimistic person and that in itself is what has kept me going throughout all the ups and downs in life." (6.) Remarkably, one aspect that emerged consistently from all participants' narratives, regardless their cultural differences, is the emphasis that they the all put on the hope for global issues to be solved. It is world peace that participants hope for (Pierina 2. 3.; Ilenia 3.), with no wars or atrocities (Gwen 2., Ilenia 4.). A world where "poverty [c] Jould be eradicated" (Gwen 2.) and where there is more justice for minority groups, including "our quite possible frail old age" (Mo 2.). When asked "what things/situations cause you to lose hope?" all participants made reference to the world situation. It seems that the realisation of their impotence (or lack of agency) in front of unresolved global issues, including the political situation, represents a significant hope-hindering factor in all eight women involved in this study. Indeed, the inability to make sense of "what's going on around the world' (Jacqui 4.) seems to be a major factor that can inhibit their capacity for hopeful thinking, despite their positive inclination towards life. Such perceived hopelessness seems to affect participants' outer faith in 'humanity'; a faith that seems to be temporarily restored when accompanied by 'symbolic collective thinking', as for Carlotta who despite "the hatred and the arrogance in the world situations" (4.), trusts "humankind because there is always a light in each of us" (6.) or Ilenia who keeps her positive attitude because she always hopes that "tomorrow all people will be united" (4.).

This type of 'universal' hope that emerged from all participants' accounts, and symbolically connected them on a collective level, could be understood as an archetypical human need for relatedness that can fall within Jung's ${ }^{19}$ theorisation of the 'collective unconscious'. Indeed, all participants shared the same desire for humankind to live in peace, almost as a way of re-conciliating their primordial image of a universal human predisposition to be united. A collective hope that is surely worth being explored further in therapeutic practices with adults in later life, and, indeed, with individuals of any age.

\section{Discussion}

The main aim of this study was to find out the meaning that hope may assume in later life, and particularly in women. The participants' hope-narratives have revealed that for them hope mainly means to be physically healthy, positive and active agents of their lives, and to have faith in self and others. Subsequently, the perceived or predicted absence of one or more of the above factors, can hinder their ability for hopeful thinking. These are surely insightful views on hope; however, it could be argued that given the genderedoriented nature of the present study, further exploration is needed in this field to also compare and contrast the views of a male sample. Participants' cultural diversity emerged to be as relatively unimportant in the current study. Nevertheless, additional studies into cognitive and emotional processes of older adults from different cultural backgrounds would surely enrich our understanding of this complex phenomenon. Indeed, hope seems to assume multi- layered meanings for each older woman involved in the study, depending on their unique external and internal responses to life experiences. On the whole, what powerfully resonates from participants' accounts, regardless their cultural differences, is an impressive amount of resilience that together with an upbeat predisposition, shared with significant others, seem to nurture their hopeful thinking also in times of physical or psychological vulnerability.

Hope is described by participants from an individual perspective, but it also emerges to be projected on a global scale. Indeed, a 'collective hope' that could derive from an ancestral need for human connectedness, seems to reunite all participants on a subliminal level. A phenomenon that can be translated in Jungian terms as part of the 'collective subconscious'. Hence, the need to explore within therapeutic practices, clients' views and perceptions of hope in its various semantic facets, expressed on a conscious and subconscious level. A 'hopeful' discourse that when reinforced by a congruent therapeutic alliance can serve as a powerful tool for clients (and therapists) to maintain or restore faith in self and humanity.

\section{Hope in practice}

The various themes in relation to hope that emerged from my participants' accounts clearly reveal that we (as mental health practitioners) need to take into account the multifaceted aspects that can foster or hinder hope in individuals. These include an appreciation of hope as a personal and universal phenomenon that when expressed and validated within therapeutic practices can help individuals age well. Given the complex aspects of hope, which also emerged from my participants' narratives, it appears to be crucial to look at this phenomenon in a holistic manner and within an integrative construct that can reflect its multidimensional often contradictory essence. Indeed, a holistic view on hope has been receiving a growing consensus among integrative psychotherapists, whose theoretical stances and practices are fundamentally relational and engaged in the present moment. Such views find fertile ground particularly in process-experiential therapies, such as person-centre ${ }^{20}$ and gestalt psychotherapy, ${ }^{21}$ and also, within psychodynamic ${ }^{15}$ and existential perspectives. ${ }^{22,23}$ Most recently, insightful views on hope have also been provided by the newly developed theoretical tradition of positive psychology, ${ }^{9}$ whose pragmatic views on therapeutic practices have inspired many cognitive behavioural psychotherapists.

As O'Hara ${ }^{4}$ suggests it is important for therapists "to be open to working in integrative and pluralistic ways, as [...] in doing so 
provides the greatest possibility for client hope to be found, ignited, or constructed" (p. 76). Indeed, as highlighted by Norcross and Goldfried, ${ }^{24}$ while there are many variables in different therapies associated with treatment effectiveness depending on assimilative and theoretical integration or technical eclecticism, hope (together with expectancies) seems to be the most important common factor that reunites all integrative psychotherapists. Particularly, when working with adults who have long-time reached maturity, as in the case of my participants, it is important to engage them in the expression of hope as one of the most powerful therapeutic interventions. ${ }^{25}$ Open narratives of hope could surely help older clients endure the multiple personal and interpersonal losses that they are likely to have encountered throughout their life. Such narratives would also nurture a renewed shared belief (from both client and therapist) that hope can be envisaged, maintained or restored in later as well as at any stage of life. ${ }^{26-29}$

\section{Acknowledgments}

None.

\section{Conflicts of interest}

The author declares that there is no conflict of interest.

\section{References}

1. Herth K. Hope in older adults in community and institutional settings. Issues in mental health nursing. 1993;14(2):139-156.

2. Herth K. Fostering hope in terminally-ill people. Journal of advanced nursing. 1990;15(11):1250-1259.

3. Eliott J, Olver I. The discursive properties of 'hope': A qualitative analysis of cancer patients' speech. Qualitative Health Research. 2002;12(2):173-193.

4. O'Hara D. Hope in counselling and psychotherapy. London: Sage. 2013.

5. Bergin L, Walsh $\mathrm{S}$. The role of hope in psychotherapy with older adults. Aging and Mental health. 2005;9(1):7-15.

6. Dufault K, Martocchio B. Hope: its spheres and dimensions. Nursing Clinics of North America. 1985;20(2):379-391.

7. Olsen SJ. Older adults in long-term care: Fostering hope amidst loss. Master of Social Work Clinical Research Papers. Paper 243. 2013.

8. Snyder CR. Handbook of hope: theory, measures, applications. New York: Academic Press. 2000.

9. Snyder CR. Hope Theory: Rainbows in the mind. Psychological Enquiry. 2002;13(4):249-275.

10. Frank JD, Frank JB. Persuasion and healing: a comparative study of psychotherapy. 3rd edition. Baltimore: Johns Hopkins Press. 1991.
11. Maslow AH. Motivation and personality. 2nd edition. In: Harper \& Row McLeod J, Editors. (2009). An introduction to counselling. London: Open University. 1970.

12. Irving LM, Snyder CR, Cheavens J, et al. The relationships between hope and outcomes at the pretreatment, beginning, and later phases of psychotherapy. Journal of Psychotherapy Integration. 2004; $14: 419-443$.

13. Sumerlin J. Self-actualisation and hope. Journal of Social Behavior and Personality. 1997;12:1101-1110.

14. Vande Kemp H. Hope in psychotherapy. Journal of Psychology and Christianity. 1984;3:27-35.

15. Jung CG. Modern man in search of soul. In: Dell WS, Baynes CF. New York: Harcourt, Brace \& World. 1933.

16. Woodyard DO. Beyond cynicism: the practice of hope. Philadelphia: Westminster. 1972.

17. Wrobleski KK, Snyder CR. Hopeful thinking in older adults: back to the future. Experimental Aging Research. 2005;31:217-233.

18. Szasz TS. The myth of mental illness: foundations of a theory of personal conduct. New York: Dell. 1961.

19. Jung CG. The Structure of the Unconscious. Collected Works vol. 7. $1916 ; 1953$.

20. Rogers C. Client-Centered Therapy: Its Current Practice, Implications and Theory. London: Constable. 1951.

21. Perls F, Hefferline R, Goodman P. Gestalt Therapy: Excitement and Growth in the Human Personality. London: Souvenir Press. 1951.

22. Kierkegaard S. Upbuilding discourses $I-I V$. In: Swendon DF \& Swendon LM, Editors. Minneapolis: Augsburg Publishing House. 1844 .

23. Marcel G. The philosophy of existentialism. In: Harari M, Editor. New York: Addison-Wesley. 1987.

24. Norcross JC, Goldfried MR. Handbook of psychotherapy integration. 2nd edition. New York: Oxford. 2002.

25. Erskine RG, Trauntman RL. Methods of an integrative psychotherapy. Transactional Analysis Journal. 1996;26(4):316-328.

26. Cooper M, Mcleod J. Pluralistic counselling and psychotherapy. London: Sage. 2011.

27. Lazarus RS. Towards better research on stress and coping. American Psychologist. 2000;55(6):665-673.

28. Oxford Dictionaries. 2018

29. Rogers C. A way of being. Boston: Houghton Mifflin. 1980. 